SHC experiences. Men and women strongly indicated willingness to wait in clinic for results if they perceived themselves at risk for infection (self-assessed as sexual risk-taking and/or having symptoms). All patients were willing to wait for AMR results following a positive result. Patient suggestions for POCT pathway implementation included: targeting POCTs to those concerned they are infected and providing information on steps and time involved for new pathways.

Conclusion Patients' willingness to wait in clinic, explained as dependent on a self-assessed risk for infection, provides nuanced understanding of patients' priorities for care. Patient suggestions that specific, directed messaging from SHCs may allow acceptability of various changes related to POCT adoption gives guidance for implementation. We recommend further research when these tests are made available, to assess these theories in practice.

\section{p4.119 HOW CAN WE IMPROVE PARTNER NOTIFICATION FOLLOWING HIV DIAGNOSIS? - A QUALITATIVE STUDY OF MEN WHO HAVE SEX WITH MEN IN MELBOURNE}

${ }^{1}$ Jane Tomnay, ${ }^{1}$ Alana Hulme-Chambers, ${ }^{2}$ Jade Bilardi, ${ }^{2}$ Christopher Fairley, ${ }^{2}$ Sarah Huffam, ${ }^{2}$ Marcus Chen. ${ }^{1}$ University Of Melbourne, Melbourne, Victoria, Australia; ${ }^{2}$ Melbourne Sexual Health Centre/Monash University, Melbourne, Victoria, Australia

\subsection{6/sextrans-2017-053264.614}

Introduction Improved partner notification (PN) following HIV diagnosis could help control HIV among men who have sex with men (MSM). However, there is little evidence exploring what this experience is like for Australian MSM and how achievable it is in the era of the internet and smartphones.

Methods MSM recently diagnosed with HIV were recruited from three health services in Melbourne for a semi-structured interview about PN experiences. Interviews were transcribed verbatim for thematic analysis using a combined deductive/ inductive approach whereby themes were derived from both previous literature, the research questions and interview schedule and inductively from emergent and recurrent themes arising from the data.

Results Three main themes arose: the fear of PN and HIV disclosure; partners' unexpected reactions; and the need for more support for PN. MSM found partner notification difficult and uncomfortable and described fear about potential repercussions of $\mathrm{PN}$. However, they felt it was the right thing to do and all partners should be notified where ever possible. Regular partners were more likely to be notified, and in person, due to the availability of contact information but more notably due to a sense of moral responsibility. Men commonly had few contact details for casual partners and preferred partner notification strategies that allowed them to remain anonymous, largely reflecting the reasons for and ways in which they met casual partners: online or through apps and predominantly for once-off, anonymous sex. Most described unexpected positive responses from partners who were contacted personally by the men.

Our study also showed that these participants required professional support to carry out PN, especially with casual partners, as well as support around understanding the implications of and treatments relating to being HIV positive.

Conclusion PN could be improved by offering more options that allow the index patient to remain anonymous, particularly when notifying casual partners.

\section{p4.120 CARE-SEEKING BEHAVIOURS AMONG HIV-INFECTED ADULTS IN MOZAMBIQUE: BARRIERS AND FACILITATORS TO TIMELY ENROLLMENT IN HIV CARE AND TREATMENT}

Amilcar Magaco. National Instituto of Health, Maputo, Mozambique

\subsection{6/sextrans-2017-053264.615}

Introduction The $\mathrm{MoH}$ of Mozambique pledged to eliminate vertical transmission, reduce sexual transmission by $50 \%$ and increase antiretroviral therapy coverage up to $80 \%$. To achieve these goals, it is essential that PLHIV must access HIV care services in a timely manner. However, HIV-infected persons in Mozambique are continuously accessing care and treatment services at an advanced stage of the disease. The goal was to determine barriers and facilitators to timely enrollment in HIV care and treatment, regardless of the disease stage.

Methods In-depth interviews were conducted to 90 newly diagnosed HIV-positive patients in one health facility in Maputo City and two urban and rural health facilities in Zambézia province. Interviews were recorded, transcribed and translated prior to analysis.

Results Long distances to the health facility, long waiting time, lack of access to transport, lack of money, and side-effects were the most frequently barriers to timely enrollment in HIV care and treatment. One of the facilitators for the timely enrollment of patients in the care and treatment of HIV is related to the fact that enrollment's process in HIV care happens the next day after the test completion. Family support associated with the basic knowledge related to HIV was mentioned as one of the main factors that led to an early onset of ART therapy. While some patients reported that the sensation of feeling good while doing the medication is one of the factors that most also contributes to ART therapy adherence, others showed to feel motivated for the treatment due to the encouragement given by their social networking.

Conclusion Adherence to treatment is influenced by the desire of feeling healthy. Our results suggest that one of the major facilitators for a timely enrollment of patients in care services and treatment of HIV is the flexibility process of enrollment in HIV care at health facilities. Side-effects are the most frequently barriers to timely enrollment in HIV care and treatment. Health providers should explain the side effects and how to handle these.

\section{p4.121 CARE-SEEKING BEHAVIOURS AMONG HIV-INFECTED ADULTS IN MOZAMBIQUE: HIV-RELATED KNOWLEDGE AND ADHERENCE TO TREATMENT}

${ }^{1}$ Reka Maulide Cane, ${ }^{1}$ Amilcar Magaco, ${ }^{1}$ Carlos Botao, ${ }^{1}$ Granelio Tamele, ${ }^{2}$ Francisco Mbofana. ${ }^{1}$ Sistemas de Saúde, Instituto Nacional de Saúde, Ministério da Saúde, Moçambique, Maputo, Mozambique; ${ }^{2}$ Direcção Nacional de Saúde Pública, Ministério da Saúde, Moçambique, Maputo, Mozambique

\subsection{6/sextrans-2017-053264.616}

Introduction The Ministry of Health of Mozambique pledged to eliminate vertical transmission, reduce sexual transmission by $50 \%$ and increase antiretroviral therapy coverage up to $80 \%$. To achieve these goals, it is essential that HIV-infected persons must access HIV care services in a timely manner. The objective of this study was to explore reasons why patients with positive diagnosis do not adhere to services and care of HIV, regardless of the disease stage. 